\title{
Synthetic yeast brews neuroactive compounds
}

\author{
Metabolic engineering offers the flexibility to meet market demand for bioactive natural products but can be \\ hampered when a necessary protein or intermediate is toxic. In yeast, modifying the subcellular localization of \\ biosynthetic enzymes can alleviate toxicity and increase production titer.
}

\section{Yang Gu and Peng Xu}

$\Lambda$ bout $70 \%$ of US Food and Drug Administration-approved drugs in the last 25 years are derived from or inspired by natural sources. The diversity and complexity of these naturally sourced compounds can offer remarkable efficacy and specificity to block host-pathogen interactions or prevent the development of critical diseases. Famous examples include the antimalarial drugs artemisinin and quinine, anticancer drugs paclitaxel and vincristine, and antimicrobial and antiviral drugs daptomycin, vancomycin, and romidepsin. However, obtaining plant-produced bioactive compounds in sufficient quantities to meet market demand is often hampered by their extremely low concentrations in their native host and their susceptibility to environmental, seasonal, and regional variations. Current plant-breeding and agricultural technologies are not sufficient to increase supply, and total chemical synthesis is not economically feasible due to the structural complexity of natural products and difficulty of their separation from co-produced analogs. As an alternative, engineering plant biosynthetic pathways in promising microbial hosts offers potentially scalable and environmentally friendly routes for the synthesis of essential drug components. Recently, a number of natural products with strong neurological activity have been engineered in yeast, including cannabinoids ${ }^{1}$, neurotransmitter inhibitor tropanes ${ }^{2,3}$, and analgesic morphine-related drugs $^{4-6}$. In this issue of Nature Chemical Biology, Grewal and Samson et al. ${ }^{6}$ report a novel approach to de-bottleneck a critical enzyme step and unlock the potential of baker's yeast (Saccharomyces cerevisiae) for the synthesis of essential analgesic drug precursors.

Metabolic engineering enables the modification of cellular reaction networks and efficient biosynthesis of desired compounds. Common strategies in pathway engineering include overexpression of rate-liming metabolic steps to increase precursor supply, elimination or attenuation of competing pathways to reduce byproduct formation, subcellular compartmentalization of specific enzymes to mitigate the dissipation of metabolic intermediates, and selection of the best chassis strain. Compared to
Escherichia coli and other bacteria, yeast has a number of favorable bioprocessing traits such as a larger cell volume (thus enabling easier cell separation), lower optimal growth temperature (requiring less cooling energy), lower cultivation $\mathrm{pH}$, stronger xenobiotic tolerance, and lower chance of phage contamination. More importantly, the spatially separated subcellular compartments in yeast provide the ideal microenvironments for specialized metabolism and functional expression of membrane-bound proteins such as cytochrome P450 enzymes ${ }^{7}$, which has proven difficult in simple prokaryotic microbes. In particular, peroxisome engineering has been extensively deployed in recent years to tailor the chain-length profiles of oleochemicals ${ }^{8}$, to use as a metabolic source for cytosolic acetyl-CoA ${ }^{9}$ and to use as a subcellular storage space for squalene overproduction ${ }^{10}$.

In their efforts to produce the analgesic drug precursor $(S)$-reticuline, Grewal and Samson et al. found that cytosolic expression of the biosynthetic pathway caused a metabolic burden that restricted production of the target compound ${ }^{6}$.

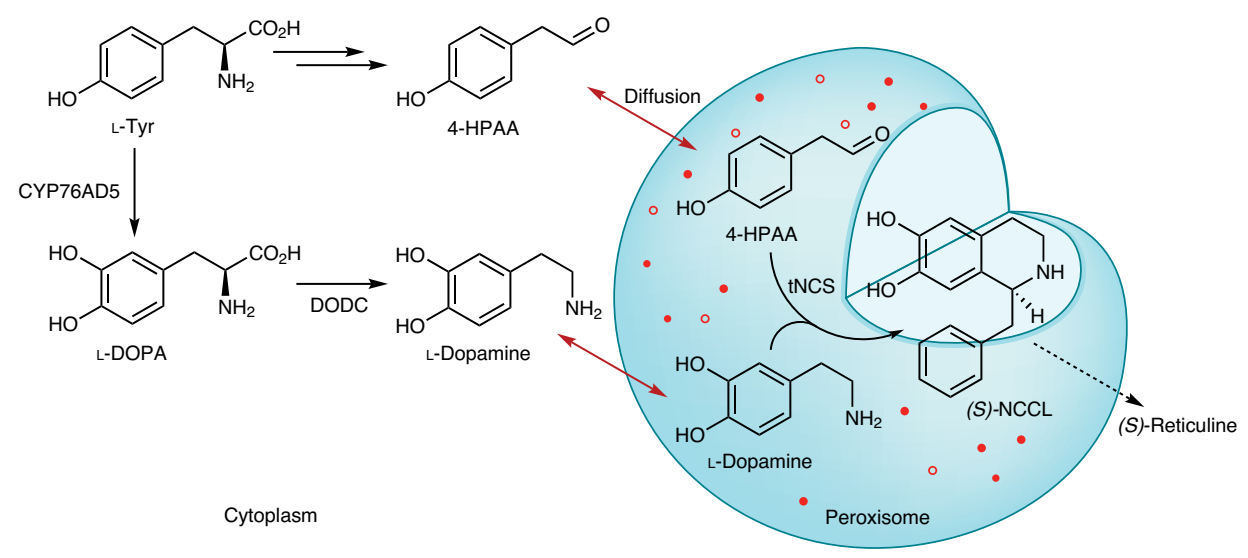

Fig. 1 | Yeast peroxisome engineering to shield enzyme toxicity and improve production titer of the analgesic precursor (S)-reticuline. Cytosolic expression of norcoclaurine synthase (NCS) causes a metabolic burden for the production host, but compartmentalization of a modified NCS into the yeast peroxisome could effectively alleviate cellular toxicity. The permeability of the peroxisome allows for free diffusion of pathway intermediates (red). L-Tyr, L-tyrosine; 4-HPAA, 4-hydroxyphenylacetaldehyde; (S)-NCCL, (S)-norcoclaurine; CYP76AD5, cytochrome P450 L-tyrosine hydroxylase; DODC, L-DOPA decarboxylase; tNCS, truncated norcoclaurine synthase with peroxisomal targeting signal. 
To troubleshoot the possible roots of this metabolic burden, the authors verified that the observed cellular toxicity is not associated with the substrates or the accumulation of products by performing a number of chemical-analog and cell-fitness studies. Instead, the toxicity was most likely derived from the expression of the critical pathway enzyme norococlaurine synthase (NCS). Traditionally, adaptive lab evaluation could be used to improve cell tolerance to a toxic metabolite or enzyme. However, this objective could not be achieved in a short period of time, so they turned to a less-explored property of the yeast peroxisome to shield enzyme toxicity by simply targeting NCS to that subcellular compartment (Fig. 1).

In cell biology, peroxisomes are characterized as the 'junk protein scavengers' that disassemble, destroy, and recycle proteins or eliminate toxic chemicals. Essential but toxic metabolic reactions or metabolites are often naturally sequestered there, protecting the rest of the cell from damaging byproducts such as lipid peroxides generated from the degradation of unsaturated fatty acids. By taking advantage of these scavenging characteristics of the yeast peroxisome, the authors successfully alleviated the pathway bottleneck caused by NCS toxicity and improved the productivity of essential benzylisoquinoline alkaloid (BIA) precursors in yeast. Considering that the molecular-weight cutoff value of metabolites passing across the peroxisome membrane is between $500-700 \mathrm{Da}$, the authors harnessed this property to facilitate passive transport of the metabolic intermediates and enable the completion of the enzymatic cascade free of diffusive limitations (Fig. 1). Furthermore, through expression of three modified transcriptional factors devoid of regulatory domains or phosphorylation sites, the authors were able to change peroxisome morphology, expanding both their surface area and total volume, thus further improving the titer of the BIA precursors $(S)$-norcoclaurine and $(S)$-reticuline.

Insulating a toxic enzyme into peroxisomes expands our toolkit to alleviate cellular toxicity, improves the catalytic efficiency of complex metabolic pathways, and offers us a valuable strategy to tailor natural product chemistry through pathway engineering. Future work on this BIA pathway may include de-bottlenecking the shikimic acid and $(S)$-adenosyl-L-methionine (SAM) precursor pathways to further improve the titer of $(S)$-reticuline. With the current challenges of COVID-19, the decline of international trade in pharmaceutical intermediates has created shortages and unstable supply chains, encouraging us to seek alternative ways of biomanufacturing to address the scarcity of essential drugs. In addition, increased incidents of social and mental-health problems have been reported, which are likely associated with anxiety, depression, chronic pain, and neurological disorders. We predict that these challenges will lead to a surging trend in the research and investment sectors to seek alternative sources of neuroactive natural products to fight pain and mental disorders.

Yang $\mathrm{Gu}^{1,2}$ and Peng Xu (D) 1 ه

${ }^{1}$ Department of Chemical, Biochemical and Environmental Engineering, University of Maryland Baltimore County, Baltimore, MD, USA. ${ }^{2}$ School of Food Science and Pharmaceutical Engineering, Nanjing Normal University, Nanjing, China.

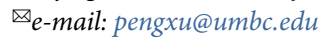

Published online: 28 October 2020 https://doi.org/10.1038/s41589-020-00691-5

References

1. Luo, X. et al. Nature 567, 123-126 (2019).

2. Srinivasan, P. \& Smolke, C. D. Nat. Commun. 10, 3634 (2019).

3. Srinivasan, P. \& Smolke, C. D. Nature 585, 614-619 (2020).

4. Li, Y. et al. Proc. Natl Acad. Sci. USA 115, E3922-E3931 (2018).

5. Pyne, M. E. et al. Nat. Commun. 11, 3337 (2020).

6. Grewal, P. S. et al. Nat. Chem. Biol. https://doi.org/10.1038/ 541589-020-00668-4 (2020).

7. Ma, J., Gu, Y. \& Xu, P. Curr. Opin. Biotechnol. 66, 140-149 (2020)

8. Xu, P., Qiao, K., Ahn, W. S. \& Stephanopoulos, G. Proc. Natl Acad. Sci. USA 113, 10848-10853 (2016).

9. Marsafari, M. \& Xu, P. Metab. Eng. Commun. 10, e00121 (2020).

10. Liu, G. S. et al. Metab. Eng. 57, 151-161 (2020).

Competing interests

There authors declare no competing interests. 